\title{
Off spells and dyskinesias: Pharmacologic management of motor complications
}

\begin{abstract}
There are two major causes of disability in patients with Parkinson disease: motor fluctuations that occur when a dose of levodopa becomes ineffective, leading to a "wearing off," and hyperkinetic movements (dyskinesias) caused by excessive levels of dopamine. The utility of continuous levodopa treatment is therefore limited by motor complications. Pharmacologic options to treat wearing off include adding (or increasing the dosage of) levodopa, adding (or increasing the dosage of) a dopamine agonist, or adjunctive treatment with a monoamine oxidase inhibitor or catechol-O-methyltransferase inhibitor. Dyskinesias will respond to a reduction in levodopa dosage at the expense of worsening parkinsonism and an increase in the number of "off" episodes. Continuous dopamine stimulation may overcome the pulsatile stimulation of postsynaptic dopamine receptors produced by standard oral formulations of levodopa that lead to motor complications.
\end{abstract}

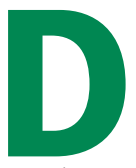

opaminergic treatment is extremely beneficial in inducing symptom improvement in early Parkinson disease (PD). Patients typically experience a smooth and even response to the early stages of levodopa treatment. With disease progression, however, the effect of levodopa begins to weaken approximately 4 hours after each dose, leaving patients anticipating the need for their next dose and vulnerable to motor fluctuations and dyskinesias.

Motor fluctuations refer to the unanticipated loss of effect of a given dose of levodopa; instead of a smooth, predictable symptomatic benefit, the patient may lose benefit earlier than usual (termed "wearing off") or may suddenly switch from "on" (symptoms controlled) to

Dr. Khan reported that she has no financial interests or relationships that pose a potential conflict of interest with this article.

This article is based on Dr. Khan's presentation at "The Annual Therapy Symposium on Movement Disorders for the Modern Clinician" held in Fort Lauderdale, Florida, on January 29, 2011. The article was drafted by Cleveland Clinic Journal of Medicine and was then reviewed, revised, and approved by Dr. Khan.

doi:10.3949/ccjm.79.s2a.02 "off” (symptoms return). Dyskinesias, or involuntary movements, occur when dopamine levels are too high.

Motor complications are a major cause of disability in PD. They affect $60 \%$ to $90 \%$ of PD patients after 5 to 10 years of treatment. Moreover, in one study of 143 PD patients, motor complications diminished quality of life; the most strongly affected dimensions were mobility, activities of daily living, communication, and stigma. ${ }^{1}$

\section{PATHOGENESIS OF MOTOR COMPLICATIONS}

Under physiologic conditions, dopamine stimulation of the striatal dopamine receptors occurs in a sustained fashion. In early PD, the pool of remaining neurons of the substantia nigra is believed to be sufficiently active to smooth out changes in levodopa levels, providing a relatively constant amount of dopamine. Many PD patients therefore have several years of trouble-free treatment following diagnosis. In the advanced disease states, however, the number of presynaptic dopaminergic neurons progressively decreases. With fewer dopaminergic neurons, a constant dopaminergic concentration cannot be sustained. As PD advances, the progressive loss of dopaminergic neurons leads to impaired dopamine storage. Thus, the buffering capacity of dopaminergic neurons decreases and synaptic dopamine levels begin to reflect systemic or exogenous levodopa levels.

With disease progression, the "honeymoon" phase diminishes, and most PD patients begin to develop motor complications after 5 or more years. At this stage, medications frequently need to be adjusted, which can be a complex task for the physician and patient. The schema for the pathogenesis of motor complications related to the disease process and chronic levodopa treatment are depicted in the Figure.

According to current views, the total motor response to levodopa results from the combination of endogenous dopamine production along with the short-duration response (SDR) and the long-duration response (LDR) to exogenous levodopa. ${ }^{2}$ The SDR represents an improvement in parkinsonian symptoms and signs, lasting minutes to hours, that is closely related to the rise and fall of plasma levodopa concentrations. The SDR parallels the fluctuations in motor response and has received 
the most attention in the literature. The LDR is an improvement in parkinsonism that builds up over days and likewise decays over days. The LDR decays more rapidly in severely affected patients. Negative response, or "super off," is a transient worsening of motor function to below the baseline level that may occur as the effects of the SDR dissipate.

The proportions of the SDR and LDR can vary according to disease progression. The LDR is more prominent in early stages, accounting for the stable response seen in the honeymoon period of treatment.

\section{Peripheral factors}

Additional peripheral factors such as changes in gastric motility and absorption contribute to motor complications. Levodopa is transported by a saturable active transporter system, the large neutral amino acid system, in the gut, and across the blood-brain barrier. Levodopa absorption is thus affected by food intake, especially protein. Levodopa and dietary amino acids compete with each other for absorption at the intestinal and blood-brain levels. Levodopa and other dopaminergic therapies further chronically reduce gastric emptying.

\section{Pulsatile dopamine stimulation}

The latency from the time of levodopa administration to the onset of motor improvement is typically 30 to 90 minutes. Latency is longer in late stages when the striatal buffer is weakened and the plasma concentration of levodopa fluctuates.

\section{TYPES OF MOTOR FLUCTUATIONS}

Fluctuating motor response in levodopa-treated patients refers to clinically apparent oscillations in motor function. Management of the fluctuating response may require frequent daily dosing of levodopa.

Motor fluctuations in PD take four forms: wearing off, off, delayed on/no on, and dyskinesias.

\section{Wearing off}

Wearing off refers to the premature loss of benefit from a given dose of levodopa, causing a predictable return of parkinsonian symptoms (bradykinesia, tremors, rigidity, and gait problems) in advance of the next scheduled dose. Observed in early and moderate PD, wearing off is the most common type of motor fluctuation. Its pathophysiology relates to disease progression and pharmacokinetics of levodopa. It can be sudden or gradual, predictable or unpredictable.

\section{Off state}

The off state is the unpredictable reappearance of parkinsonian symptoms at a time when central levels of antiparkinsonian drugs are expected to be within the target therapeutic range. Such symptoms include
Pathogenesis of motor complications: Summary
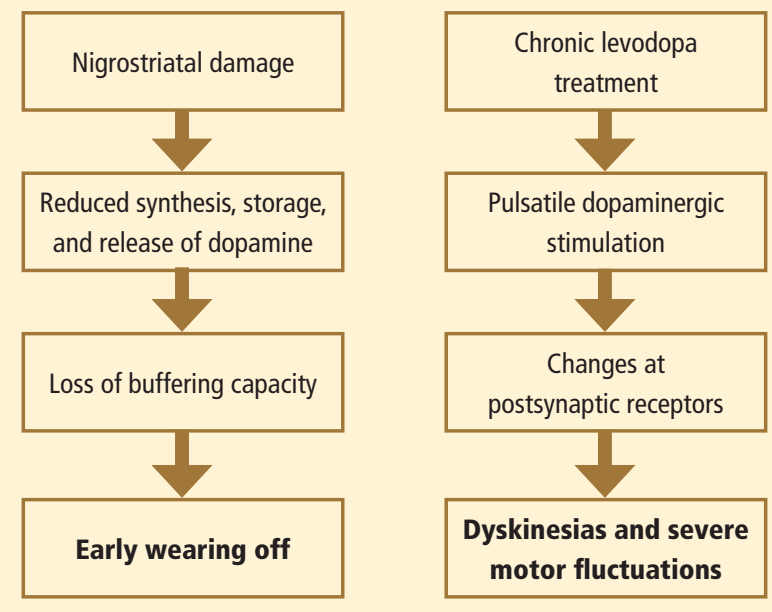

FIGURE. Pathogenesis of motor complications related to the Parkinson disease (PD) process and levodopa therapy. As PD advances (left), the progressive loss of dopaminergic neurons associated with nigrostriatal damage leads to impaired dopamine storage and clearance. This reduces the buffering capacity of dopaminergic neurons, causing early wearing off. During chronic levodopa treatment (right), pulsatile dopaminergic stimulation causes changes in postsynaptic receptors, referred to as "priming," that increase the responsiveness of the receptors. The increased response results in severe levodopainduced dyskinesias and on-off fluctuations. These postsynaptic changes are mediated through postsynaptic dopamine $D_{1}$ receptors and $\mathrm{N}$-methyl-D-aspartate glutamate receptors.

pain, stiffness, paresthesia, cognitive symptoms (depression, anxiety, difficulty with concentration, and mental slowing), inner restlessness, and inner tremulousness. The off state can be sudden or gradual, predictable or unpredictable.

\section{Delayed on/no on}

Delayed on is a prolongation of the time required for the central antiparkinsonian drug effect to appear. As the disease progresses, wearing off becomes more complicated and more unpredictable. The dosing responses vary, and patients sometimes report delayed on. The causes of delayed on or no on can be an insufficient dose, dosing with high-protein meals, or delayed gastric emptying. Metoclopramide or domperidone can help with gastric emptying. Metoclopramide can cross the blood-brain barrier and thus may cause adverse effects related to dopaminergic blockade; domperidone does not cross the blood-brain barrier.

\section{Dyskinesias}

Dyskinesias are hyperkinetic movements related to dopaminergic effects that are greater or less than the therapeutic threshold. They are common with long-term 
TABLE

Mechanisms for treatment of off and wearing off

\begin{tabular}{|c|c|c|}
\hline Drugs & Mechanism & Comments \\
\hline $\begin{array}{l}\text { MAO-B inhibitors } \\
\text { (rasagiline, selegiline, } \\
\text { Zydis selegiline) }\end{array}$ & $\begin{array}{l}\text { Inhibit a central dopamine catabolic } \\
\text { pathway to prolong the half-life of } \\
\text { dopamine }\end{array}$ & $\begin{array}{l}\text { Increase on time, decrease off time; } \\
\text { can induce serotonin syndrome if } \\
\text { used with tricyclic antidepressants } \\
\text { or SSRls }\end{array}$ \\
\hline $\begin{array}{l}\text { COMT inhibitors } \\
\text { (entacapone, } \\
\text { tolcapone) }\end{array}$ & $\begin{array}{l}\text { Block peripheral degradation of } \\
\text { levodopa; prolong half-life and } \\
\text { availability; tolcapone also blocks } \\
\text { central degradation }\end{array}$ & $\begin{array}{l}\text { Increase daily on time; tolcapone } \\
\text { significantly improves off time, but } \\
\text { risk of liver impairment requires } \\
\text { monitoring every other week }\end{array}$ \\
\hline $\begin{array}{l}\text { Controlled-release } \\
\text { levodopa }\end{array}$ & $\begin{array}{l}\text { Provides more constant delivery of } \\
\text { levodopa to the striatum }\end{array}$ & $\begin{array}{l}\text { Variable absorption; more effective } \\
\text { in patients with less severe } \\
\text { wearing off }\end{array}$ \\
\hline $\begin{array}{l}\text { Dopamine agonists } \\
\text { (pramipexole, } \\
\text { ropinirole, } \\
\text { apomorphine, } \\
\text { bromocriptine) }\end{array}$ & $\begin{array}{l}\text { Directly stimulate dopamine } \\
\text { receptors }\end{array}$ & $\begin{array}{l}\text { Adjunctive therapy that reduces } \\
\text { wearing off; must be discontinued } \\
\text { at first sign of side effects: ankle } \\
\text { edema, hallucinations, somnolence, } \\
\text { impulse control disorders }\end{array}$ \\
\hline
\end{tabular}

COMT = catechol-0-methyltransferase; $\mathrm{MAO}=$ monoamine oxidase inhibitor; $\mathrm{SSRI}=$ selective serotonin receptor inhibitor

\section{Food and tolerance}

The patient should not take levodopa with proteincontaining meals, particularly if his or her PD is at an advanced stage. If excessive nausea, vomiting, or lightheadedness prevents the patient from taking an adequate dose, adding carbidopa (up to $75 \mathrm{mg}$ ) to the regimen will be helpful.

\section{MAO-B inhibitors}

By inhibiting one of the central dopamine catabolic pathways, MAO-B inhibitors (selegiline, rasagiline, and Zydis selegiline) prolong the half-life of dopamine in the brain and increase on time.

Improvement in off time with rasagiline is comparable to that seen with the

levodopa therapy and have three patterns:

Off dystonia occurs when levodopa concentrations are low and the SDR has dissipated. Dystonic states may be a manifestation of too little or too much levodopa; differentiating the two is important. Off dystonia occurs mostly in the early mornings, when plasma levodopa levels are low, and mostly involves the more affected side first.

Peak-dose dyskinesia, which occurs during the SDR, is the most common type of dykinesia and is related to peak plasma levodopa levels. It is characterized by stereotypic, choreic abnormal movements involving the head, neck, trunk, and limbs, and possibly hemidyskinesia in young-onset PD. Peak-dose dyskinesias are sometimes severe enough to be disabling.

Diphasic dyskinesias are stereotyped, dystonic, or choreic movements that occur at the beginning of the SDR and again as the SDR dissipates. They predominantly affect the legs and spare the trunk, neck, and arms.

\section{TREATMENT OF OFF AND WEARING OFF}

Increasing dopaminergic stimulation is the backbone of treatment of off periods or wearing off. The strategies to increase dopaminergic stimulation include addressing food and tolerance issues and adding a monoamine oxidase $\mathrm{B}(\mathrm{MAO}-\mathrm{B})$ inhibitor or a catechol-O-methyltransferase (COMT) inhibitor such as entacapone or tolcapone to the regimen (Table). If the patient is already taking levodopa or a dopamine agonist, the dosage can be increased; or, levodopa can be added to a dopamine agonist regimen and vice versa.
COMT inhibitor entacapone. In an 18-week, doubleblind trial of 687 patients randomized to receive oncedaily rasagiline, entacapone, or placebo as an adjunct to levodopa, both rasagiline and entacapone reduced off time by 1.2 hours compared with placebo. ${ }^{3}$

In a 26-week placebo-controlled study, rasagiline decreased off time by $29 \%$ when added to levodopa in patients with PD and motor fluctuations, compared with a $15 \%$ reduction in the placebo group. ${ }^{4}$ This study confirmed the benefit of adding rasagiline to the regimens of patients who were already optimally treated with levodopa, dopamine agonists, amantadine, anticholinergics, and entacapone before enrolling in the study.

An orally disintegrating selegiline (Zydis selegiline) tablet is particularly useful for patients who have difficulty swallowing. The bioavailability of Zydis selegiline is $80 \%$ compared with $10 \%$ for selegiline, resulting in faster absorption. Pregastric absorption of Zydis selegiline avoids extensive first-pass metabolism in the liver and, therefore, the concentration of amphetamine-like metabolites is much lower.

In a 3-month, placebo-controlled study of patients with PD who were experiencing levodopa-related motor fluctuations, Zydis selegiline was associated with a 2.2-hour reduction in the total number of off hours compared with 0.6 hours in the placebo group, and dyskinesia-free on hours increased by 1.8 hours. ${ }^{5}$

The use of MAO-B inhibitors with tricyclic antidepressants or selective serotonin reuptake inhibitors has been reported to induce the serotonin syndrome by 
activation of $5 \mathrm{HT}_{1 \mathrm{a}}$ and $5 \mathrm{HT}_{2}$ receptors. Serotonin syndrome is a potentially life-threatening accumulation of serotonin that can cause encephalopathy, severe rigidity of the legs, dysautonomia (diarrhea, mydriasis, and excessive lacrimation), myoclonus, hyperreflexia, and seizures.

\section{COMT inhibitors}

Catechol-O-methyltransferase inhibitors (entacapone and tolcapone) block peripheral degradation of levodopa. Tolcapone also blocks central degradation of levodopa and dopamine. These mechanisms increase central levodopa and dopamine levels and prolong levodopa half-life and bioavailability. Tolcapone has more powerful COMT inhibition than entacapone because tolcapone crosses the blood-brain barrier and inhibits the peripheral and central pathways of levodopa degradation. Use of COMT inhibitors can increase daily on time, but diarrhea is a common side effect and leads to withdrawal of these agents in about 3\% of patients.

Tolcapone-treated patients show significant improvement in off time with improvement in motor fluctuations. ${ }^{6}$ Because tolcapone causes rare instances of fulminant hepatitis, liver function needs to be monitored every other week. For this reason, tolcapone should be reserved for patients in whom other treatments, including entacapone, have failed.

\section{Controlled-release levodopa}

Controlled-release levodopa was developed to provide more constant delivery of levodopa to the striatum. The benefit of controlled-release levodopa is only mild, however, as absorption of this formulation is variable. In advanced PD cases, the effects of controlled-release levodopa are more unpredictable than those with standard levodopa. Controlled-release levodopa is effective in patients with less severe wearing off, but it is not as effective in patients with a less predictable pattern of fluctuations.

\section{Dopamine agonists}

Dopamine agonists (pramipexole, ropinirole, apomorphine, and bromocriptine) have shown beneficial effects as adjunctive therapy to reduce wearing off. Side effects of dopamine agonists include ankle edema, hallucinations, somnolence, and impulse control disorders. These side effects should be discussed with patients before instituting therapy, and therapy should be discontinued if any of them occur.

In patients with advanced PD, pramipexole was shown to improve motor function during on and off periods, decrease the total off time, and decrease the severity of off time. Further, a larger reduction in the dosage of levodopa was possible in the pramipexoletreated patients than in the placebo-treated patients. ${ }^{7}$
In a comparison of pramipexole with levodopa on the end point of motor complications of PD in 300 patients, the incidences of wearing off and dyskinesia were significantly lower in the patients randomized to pramipexole with follow-up over 4 years. ${ }^{8}$ Only $25 \%$ of patients initially treated with pramipexole exhibited dyskinesia compared with $54 \%$ of patients initially treated with levodopa. Forty-seven percent of patients in the pramipexole group experienced wearing off compared with $63 \%$ initially treated with levodopa. Pramipexole is available as tablets ranging from 0.125 $\mathrm{mg}$ to $1.5 \mathrm{mg}$ in size. It is given in three divided daily doses with gradual increments of $0.25 \mathrm{mg}$ three times a day every week. Pramipexole is now also available in an extended-release formulation for once-a-day dosing in tablets ranging in size from $0.375 \mathrm{mg}$ to $4.5 \mathrm{mg}$.

Ropinirole adds clinical benefit in PD patients with motor fluctuations and also permits a reduction in the dosage of levodopa. ${ }^{9}$

In one study, ropinirole monotherapy was compared with levodopa therapy in 268 patients with early PD. By the end of the 5-year study, $45 \%$ of the levodopa patients experienced dyskinesias versus 20\% of the ropinirole patients. ${ }^{10}$

Ropinirole is available as tablets ranging in size from $0.25 \mathrm{mg}$ to $5 \mathrm{mg}$. It is now also available in an extendedrelease (XL) formulation, with tablet sizes ranging from $2 \mathrm{mg}$ to $12 \mathrm{mg}$. Ropinirole XL is taken once a day.

Bromocriptine is an old ergot-derived dopamine agonist that has also been studied for monotherapy and add-on treatment in PD. Due to the potential risks of pulmonary, retroperitoneal, and heart valve fibrosis, bromocriptine is not commonly used.

Apomorphine was approved by the US Food and Drug Administration in 2004 as an acute, intermittent, subcutaneous injection for the treatment of hypomobility off episodes (end-of-dose wearing off and unpredictable on-off episodes) associated with advanced PD. Apomorphine has been shown to be beneficial in patients with unpredictable off periods. ${ }^{11}$ Its onset of action is 10 to 15 minutes, and the effects of each dose last for 60 to 90 minutes. The best tolerated dose is 4 $\mathrm{mg}$ to $10 \mathrm{mg}$. Apomorphine appears to be most useful as as rescue medication in the refractory off periods with severe bradykinesia and unpredictable off periods.

\section{TREATMENT OF DYSKINESIAS}

Reduction of levodopa doses will reduce the frequency of dyskinesias, but at a cost of worsened parkinsonism and increased numbers of off periods. An alternative is to spread out the doses of levodopa (more frequent smaller doses), but this practice has not achieved good results. Replacing levodopa with dopamine agonists can also reduce the frequency of dyskinesias, but control 
of PD symptoms is less optimal than with levodopa. Amantadine and clozapine both have been shown to reduce dyskinesias.

\section{Amantadine}

Amantadine is an N-methyl-D-aspartate antagonist with antidyskinesia effects. Metman et $\mathrm{al}^{12}$ demonstrated that amantadine reduced dyskinesia severity by $60 \%$, without exacerbation of motor function, in a randomized placebo-controlled crossover study. Dose-response studies with amantadine have not been conducted, but 100 $\mathrm{mg}$ two or three times daily is used in practice. In some studies, a short duration of benefit has been a concern. Side effects of amantadine include leg edema, hallucinations, confusion, and rash.

\section{Clozapine}

Clozapine is an atypical antipsychotic that has been shown in open-label trials and a randomized, doubleblind, placebo-controlled trial to reduce the duration and severity of levodopa-induced dyskinesias without worsening of parkinsonian features and with no change in motor fluctuation. ${ }^{13}$ No benefit of clozapine was observed during activation dyskinesia, however. Clozapine carries the inconvenience of weekly blood draws to monitor for the development of agranulocytosis, which occurs rarely.

\section{GAIT FREEZING}

Gait freezing, most commonly a manifestation of off states, causes substantial disability. It has been thought to occur as a result of a loss of noradrenaline due to locus ceruleus degeneration. Improvement in gait freezing has been shown with apomorphine and methylphenidate.

\section{CONCEPT OF CONTINUOUS DOPAMINE STIMULATION}

Short-acting dopaminergic drugs have the potential for nonphysiologic pulsatile stimulation of postsynaptic receptors, leading to motor complications. Continuous dopaminergic stimulation to prevent this pulsatile stimulation would theoretically avoid motor complications. ${ }^{14}$ Continuous dopaminergic stimulation can be achieved by using the extended-release formulation of ropinirole or pramipexole or by continuous delivery of levodopa or dopamine agonists. Several double-blind controlled trials have shown that treatment with longacting dopamine agonists lowers the risk of motor complications compared with short-acting levodopa treatment.

In 2005, Stocchi concluded that in patients with advanced PD, a continuous infusion of levodopa was more effective in reducing motor complications than standard oral formulations. ${ }^{15}$ The reduction in motor complications was attributed to avoidance of low plasma levodopa trough levels; motor complications were not affected by relatively high plasma levodopa concentrations. The authors of this study speculated that if oral levodopa could be given "in a manner that mirrors the pharmacokinetic pattern of infusion," it might be able to reduce motor complications.

This hypothesis led to an interest in treatment with levodopa plus entacapone. A regimen of levodopacarbidopa-entacapone, four times daily at 3.5-hour intervals, was compared with levodopa-carbidopa in 747 patients with early PD over 134 weeks. ${ }^{16}$ Initiating levodopa therapy with levodopa-carbidopa in combination with entacapone did not delay the induction of dyskinesia compared with levodopa-carbidopa alone. In fact, levodopa-carbidopa-entacapone was associated with a shorter time to onset and an increased frequency of dyskinesia compared with levodopa-carbidopa.

\section{Potential future treatment options}

An intrajejunal pump system delivers a constant-rate infusion of levodopa. A double-blind study of this system is being conducted in the United States. Implantation of the system is an invasive procedure with the potential for infection, kinking dislocation, and occlusion and reposition of the catheter.

Miniature pumps for continuous subcutaneous delivery of apomorphine, currently available only in Europe, have been shown to reverse dyskinesias and motor fluctuations. Limitations of the minipumps are the development of red itchy nodules, ulcerations, and abscesses at infusion sites.

\section{Extended-release dopamine agonists}

Extended-release formulations of the dopamine agonists ropinirole and pramipexole are easy to administer, and they maintain therapeutic plasma levels for up to 24 hours. They are unlikely to replace stronger continuous dopamine stimulation with levodopa and apomorphine.

\section{SUMMARY}

Motor complications in PD result from progression of the disease and limitations of levodopa. Although the effects of levodopa on PD eventually wane, leaving patients vulnerable to motor complications, clinicians should not undertreat patients.

Effective options for the management of motor complications include prolonging the efficacy of levodopa through the use of selective MAO-B inhibitors and COMT inhibitors as adjuncts to levodopa or continuous dopaminergic stimulation achieved by the use of longacting dopamine agonists or continuous intraduodenal levodopa.

Emerging therapies will be more efficient for continuous delivery of dopaminergic drugs. Pump delivery systems and extended-release formulations have shown promise. 


\section{REFERENCES}

1. Chapuis S, Ouchchane L, Metz O, Gerbaud L, Durif F. Impact of the motor complications of Parkinson's disease on the quality of life. Mov Disord 2005; 20:224-230.

2. Nutt JG, Holford NHG. The response to levodopa in Parkinson's disease: imposing pharmacological law and order. Ann Neurol 1996; 39:561-573.

3. Rascol O, Brooks DJ, Oertel W, et al. Rasagiline as an adjunct to levodopa in patients with Parkinson's disease and motor fluctuations (LARGO, Lasting effect in Adjunct therapy with Rasagiline Given Once daily, study): a randomised, double-blind, parallel-group trial. Lancet 2005; 365:947-954.

4. Parkinson Study Group. A randomized placebo-controlled trial of rasagiline in levodopa-treated patients with Parkinson disease and motor fluctuations: the PRESTO study. Arch Neurol 2005; 62:241-248.

5. Waters CH, Sethi KD, Hauser RA, et al. Zydis selegiline reduces off time in Parkinson's disease patients with motor fluctuations: a 3-month, randomized, placebo-controlled study. Mov Disord 2004; 19:426-432.

6. Rajput AH, Martin W, Saint-Hilaire MH, Dorflinger E, Pedder S. Tolcapone improves motor function in parkinsonian patients with the "wearing-off" phenomenon: a double-blind, placebo-controlled, multicenter trial. Neurology 1997; 49:1066-1071.

7. Lieberman A, Ranhosky A, Korts D. Clinical evaluation of pramipexole in advanced Parkinson's disease: results of a doubleblind, placebo-controlled, parallel-group study. Neurology 1997; 49:162-168.

8. Parkinson Study Group. Pramipexole vs levodopa as initial treatment for Parkinson disease: a 4-year randomized controlled trial. JAMA 2004; 61:1044-1053.
9. Lieberman A, Olanow CW, Sethi K, et al. A multicenter trial of ropinirole as adjunct treatment for Parkinson's disease. Neurology 1998; 51:1057-1062.

10. Rascol O, Brooks DJ, Korczyn AD, et al. A five-year study of the incidence of dyskinesia in patients with early Parkinson's disease who were treated with ropinirole or levodopa. N Engl J Med 2000; 342:1484-1491.

11. Dewey RB Jr, Hutton JT, LeWitt PA, Factor SA. A randomized, double-blind, placebo-controlled trial of subcutaneously injected apomorphine for parkinsonian off-state events. Arch Neurol 2001; 58:1385-1392.

12. Metman LV, Del Dotto P, van den Munckhof P, Fang J, Mouradian MM, Chase TN. Amantadine as treatment for dyskinesias and motor fluctuations in Parkinson's disease. Neurology 1998; 50:1323-1326.

13. Durif F, Debilly B, Galitzky M, et al. Clozapine improves dyskinesias in Parkinson disease: a double-blind, placebo-controlled study. Neurology 2004; 62:381-388.

14. Olanow CW, Obeso JA, Stocchi F. Continuous dopamine-receptor treatment of Parkinson's disease: scientific rationale and clinical implications. Lancet Neurol 2006; 5:677-687.

15. Stocchi F, Vacca L, Ruggieri S, Olanow CW. Intermittent vs continuous levodopa administration in patients with advanced Parkinson disease: a clinical and pharmacokinetic study. Arch Neurol 2005; 62:905-910

16. Stocchi F, Rascol O, Kieburtz K, et al. Initiating levodopa/carbidopa therapy with and without entacapone in early Parkinson disease: The STRIDE-PD study. Ann Neurol 2010; 68:18-27.

Correspondence: Tarannum S. Khan, MD, Dept. of Neurology, Cleveland Clinic Florida, 2950 Cleveland Clinic Blvd., Weston, FL 33331; khant@ccf.org 